\title{
A REVIEW: RECOGNIZING CLOTHES PATTERNS AND COLOURS FOR BLIND PEOPLE USING NEURAL NETWORK
}

\author{
Shweta Joshi \\ Department of Electronics and Communication Engineering \\ Aravali Institute of Technical Studies \\ Udaipur, India
}

\author{
Aabhas Mathur \\ Associate professor \\ Department of Electronics and Communication Engineering \\ Aravali Institute of Technical Studies \\ Udaipur, India
}

\author{
Dr. Hemant Dhabai \\ Director \\ Aravali Institute of Technical Studies \\ Udaipur, India
}

\begin{abstract}
In this paper, the system of automatically recognizing clothes pattern and colors is proposed. The image is captured by the camera and get processed to identify the pattern of the clothes that is chosen. This can be classified using the support vector machine algorithm. For this the features of the image have to be obtained. These features can be extracted using three descriptors. The Radon Signature descriptor is to extract statistical properties, the wavelet subbands are used to extract global features of clothing patterns. This gets combined with local features that are obtained from scale invariance feature transform to recognize complex clothing patterns. After identifying the features the support vector machine classify the images in order to their categories. The system uses the CCNY Clothing Pattern dataset. This system can be an effective method for all the visually impaired people that they can identify the pattern and respective colors without any help.
\end{abstract}

Keywords: support vector machine algorithm, Radon signature descriptor, scale invariance feature transform, CCNY.

\section{INTRODUCTION}

The eye is one of the most complex and important sensory organs in the human body. They provide us vision, the ability to process visual detail, as well as enabling several photo response functions that are independent of vision. Eyes detect light and convert it into electro-chemical impulses in neurons. Its three types of cone cells are able to distinguish millions of distinct colour and produce the high quality images that the body relies on for many daily activities. This primary sense organ also helps us to identify and wear clothes of suitable pattern and colour which is very much important in modern day society. But what about blind people. According to WHO (World Health Organization) 285 million people are estimated to be visually impaired all over the world. And about $90 \%$ of the world's visually impaired live in low-income settings. For visually blind people selection of clothes with suitable colour and pattern becomes a tough task for them. They can manage this difficulty with the help of other people. Some of them use plastic Braille labels or different type of electronic assistance but they cost high[4].

Image processing is a method to convert an image into digital form and perform some operations on it, in order to get an enhanced image or to extract some useful information from it. It is a type of signal dispensation in which input is image, like video frame or photograph and output may be image or characteristics associated with that image. Usually Image Processing system includes treating images as two dimensional signals while applying already set signal processing methods to them. It is among rapidly growing technologies today, with its applications in various aspects of a business[3]. Image Processing forms core research area within engineering and computer science disciplines too.

To overcome the problems of visually impaired people of identifying and selecting a suitable cloth pattern and colour for them the technique of image processing can be very much helpful[1],[2]. In [4] a computer vision based system is developed to recognize clothing pattern in four categories of pattern and identify colour. In [8] and [9] the texture was identified, but finding the texture with very less dataset is not useful because the intensity value and directionality changes for all the images, so the local feature is to be extracted to overcome this problem. Due to large variance and local points of the same clothing pattern categories, global features and directionality of clothing patterns are stable within the same category. Therefore, it is able to obtain best result with local feature extraction. The combination of global and local features extraction for clothing pattern recognition that is radon Signature, Statistical descriptor (STA) and scale invariant feature transform (SIFT)[4].

\section{A. Training and testing images}

The clothing patterns can be comes under the four categories they are 
1. Irregular

2. Pattern less

3. Plaid

4. Stripe
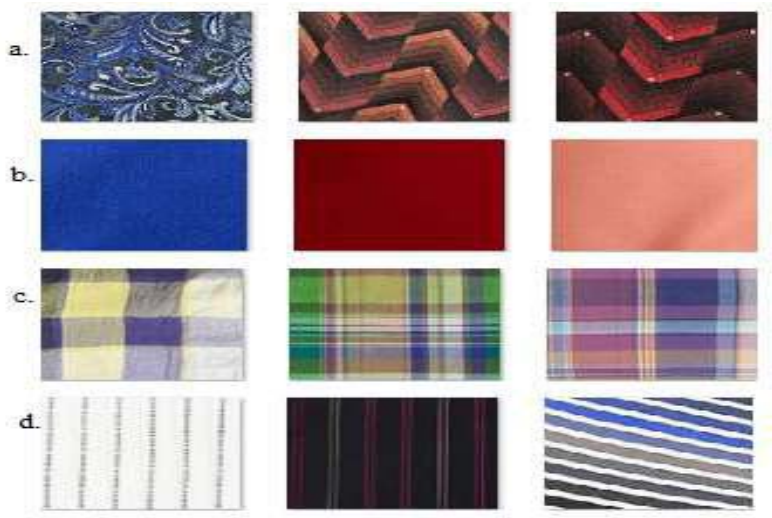

Fig 1: Dataset of training image

The entire clothing pattern can be inside this vast datasets of CCYN. Each one pattern has its own directionality, intensity and lighting variation. If the test image of stripe pattern the image patches are horizontal direction and but in the training set the image patches are in vertical direction. This can be matched by rotation, illumination changes. This adjustment can be done only by extracting the global features like energy, entropy, variance, uniformity. Once the superlative function has been estimated according to the particular image, every pixel in the image is mapped in the same way, independent of the value of surrounding pixels in the image. These techniques are simple and fast, but they can cause a loss of contrast. Examples of common global tone mapping methods are contrast reduction. Local features are the points, small patches and lines. This two features combined together to get the position of each image pixels. These pixels can be in the matrix form. So they combined together using the classifier[1].

\section{LITRATURE REVIEW}

IEEE in its $2^{\text {nd }}$ International Conference on Innovations in Information Embedded and Communication Systems 2015 published Jarin Joe Rini J, Thilagavathi B's paper "Recognizing clothes patterns and colours for blind people using neural network". In this paper the system for atomatic recognization of cloth pattern and colour was proposed. The system uses the CCNY Clothing Pattern dataset. This system can be an effective method for all the visually impaired people that they can identify the pattern and respective colors without any help. The recognizing process depends basically on two factors they are preprocessing and feature extraction they are required to implement a system to recognize the different patterns. For that the training algorithm called support vector machine are used. The figure 2 gives the flow diagram of the system[1].

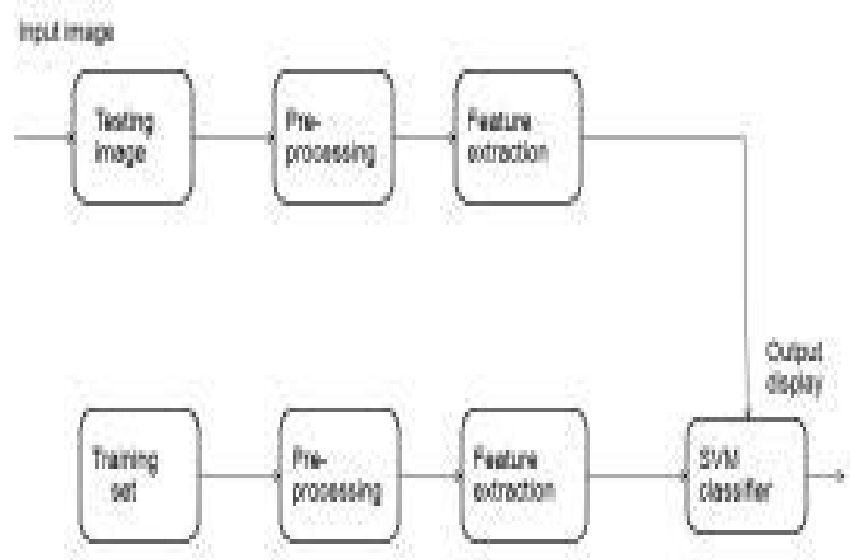

Fig.2.Block diagram

The system uses the dataset of CCNY, which dataset includes 627 images of four different typical clothing pattern designs are plaid, striped, patternless, and irregular. All the images are trained and given to the SVM algorithm[4].

Mrs. Anuradha.S.G, Thogaricheti Ashwini, “Clothing Color and Pattern Recognition for Impaired people”. In this paper published in International Journal Of Engineering And Computer Science in 2016 a study of Human-ComputerInteraction (HCI) the design and use of technology using digitalized computer systems mainly focusing on the particular interfaces between people and computers has been done. This system mainly introduces the thematic study on "Blind and visually impaired people Human computer and access to Graphics” represents a current research study towards solution for impaired people and brings together a new researchers and practitioners[5]. The Raspberry Pi board or a ARM board will be loaded with voice recognition library, Hence when user says a word the library is programmed to recognize the a word from user's voice. Based on the pronounced word appropriate action is taken[5].

International Journal of Science and Research (IJSR) in 2013 published Manisha Dhongade's paper a study on "Clothing Pattern Recognition for Blind using SURF and combined GLCM, Wavelet” where a system to take care for blind persons problem of recognizing clothing pattern and colour[10]. In this paper literature survey on texture analysis methods and assistive techniques for blind people is discussed. Feature extraction for pattern recognition is included in section[6]. The details of Clothing color and pattern identification are demonstrated. SURF (speedup robust features) an algorithm in computer vision to detect and describe local features in images has been used. The algorithm was published by H. Bay[10], in 2006 . The proposed clothing patterns and colors recognition system would help visually impaired people in their daily life. Color detection is based on normalized color in HSV color space and is able to detect multiple colors including red, black, orange, yellow, green, grey, cyan, blue, purple, pink, and white. A method to classify clothes patterns into 4 categories: striped, checks, plain and patternless are 
proposed here. The method provides new functions to improve the life quality for blind[6].

Reshmi Abraham, L. K Joshila Grace’s paper “A Texture Extraction Technique for Cloth Pattern Identification" published in Contemporary Engineering Sciences in year 2015. The main aim of the research was to increase the efficiency of indexing and image retrieval performance and also increase the similarity distance computation in cloth pattern identification[7]. The proposed system contributes in three directions. Initially, the images are trained along with the audio and text description. At first the proposed system use color based feature extraction to extract the local and global colour features. These extracted features are grouped or combined by using SVM classifier and generate the pattern. The pattern is recognized by using Texture estimation algorithm. Here, each pixel is divided into backbone blocks and perform solid colour estimation and uniform colour estimation to extract the texture of the image. Finally, extracted texture of trained image and tested image is compared and respective sound and text file is displayed to the user[13].

\section{AUTHOR'S REVIEW}

We have inspected above papers and inferred that recognizing cloth pattern and colour is a promising innovation for further research and fundamental. The system method provides a simple and reliable method for recognizing the pattern and the colour. The images are taken from CCNY database in order to implement the proposed system. All data were pre-processed and the feature of the images can be distinguished. This development of automatic recognizing clothing pattern system capable of real time identification of the pattern and the colour in the cloth. In this project the image captured by the camera and get processed to identify the pattern of the clothes that is chosen. This system is more efficient and accurate to recognize the patterns because it can classify with large number of dataset number of operations by reducing number of samples to be processed which results in reduced processing time required to be processed[12]. The future work includes implementation of automatic recognizing system in processor in order to implement the recognizing in embedded system. This system is validated in a raspberry pi processor[11]. Though being an excellent technology it has shortcomings. As the cloth pattern and colour is still displayed as text on screen, the blinds still are dependent on others.

\section{PROPOSED WORK}

Our proposal is to design \& development of an advanced clothing pattern recognition system for aiding the visually impaired using real time video processing techniques. Artificial Intelligence can be used in conjunction with proven data analysis/classification techniques such as KMeans Clustering or SVM (Support Vector Machine), to improve the accuracy of existing systems. Real Time Speech Synthesis or Text to Speech Interface can be implemented, so various pattern header \& information can be spoken by system in real time. Modular design of the system will allow reproduction of the MATLAB Simulated system into real world standalone hardware platforms with Digital Signal Processing capabilities. There is possibility of implementation of this entire system on the low cost, credit card sized computer, Raspberry Pi. There is also Possibility of induction of face recognition system in combination with existing system, to identify the person also along with clothing pattern and color. Augmentation of additional detection features such as Hair Style, Accessories, Lip Color/Lip Stick, Spectacles Etc. can be done.

\section{ACKNOWLEDGEMENT}

First and foremost praises and thanks to the God, the Almighty, for His showers of blessings throughout my research work to complete the review study successfully. I would like to express my deep and sincere gratitude to my research supervisor Mr. Aabhas Mathur Associate Professor Department of Electronics and Communication for giving me the opportunity to do this study and providing invaluable guidance throughout this review. I would also like to thank Mr. Hemant Dhabai (Director, Aravali Institute of Technical Studies) for his valuable support and guidance. I am extremely grateful to my parents for their love, prayers, caring and sacrifices for educating and preparing me for my future.

\section{REFERENCES}

[1] L. Davis, S. Johns, and J. Aggarwal, "Texture analysis using generalized co-occurrence matrices," IEEE Trans. Pattern Anal. Mach. Intell., vol. PAMI-1, no. 3, pp. 251-259, Jul. 1979.

[2] Wang Z. and J. Yong, "Texture Analysis and Classification with Linear Regression Model based on Wavelet Transform," IEEE Trans. on Image Processing.

[3] A.Arditi and Y.Tian, "User interface preferences in the design of a camera based navigation and way finding aid,” J. Visual Impairment Blindness, vol. 107, no. 2, pp. 18-129, 2013.

[4] Jarin Joe Rini J, Thilagavathi B, Recognizing clothes patterns and colours for blind people using neural network, IEEE Sponsored 2nd International Conference on Innovations in Information Embedded and Communication Systems ( ICIIECS'15).

[5] Mrs. Anuradha.S.G, Thogaricheti Ashwini, Clothing Color and Pattern Recognition for Impaired people, International Journal Of Engineering And Computer Science ISSN: 23197242 Volume 5 Issue 4 April 2016, Page No. 16317-16324.

[6] Manisha Dhongade, Clothing Pattern Recognition for Blind using SURF and combined GLCM, Wavelet, International Journal of Science and Research (IJSR) ISSN (Online): 23197064 Index Copernicus Value (2013): 6.14 | Impact Factor (2013): 4.438.

[7] Reshmi Abraham, L. K Joshila Grace, A Texture Extraction Technique for Cloth Pattern Identification, Contemporary Engineering Sciences, Vol. 8, 2015, no. 3, 103 - 108 HIKARI Ltd.

[8] Jarbas Joaci de Mesquita Sa, Andre Ricardo Backes,Paulo Cesar Cortez (2013) "Texture analysis and classification using shortest paths in graphs”, Elsevier, pattern recognition, 1314-1319.

[9] Xiaodong Yang, YingLi Tian (2013) "Texture representations using subspace embeddings", Elsevier, pattern recognition, 1130-1137. 
[10] Herbert Bay, Tinne Tuytelaars, and Luc Van Gool. "Surf: Speeded up robust features," in Computer Vision-ECCV 2006, pages 404-417. Springer, 2006.

[11] Real-Time Detection and Reading of LED/LCD Displays for Visually Impaired Persons”, E. Tekin, J. Coughlan, and H. Shen, in Proc. IEEE Workshop Appl. Comput. Vision, 2011, pp. 491-496.

[12] J. Rose, “Closet buddy: dressing the visually impaired,” In Proceedings of the 44th annual Southeast regional conference, pages 611-615. ACM, 2006.
[13] Hodges, B., Burton, D., \& Uslan, M. 1407. Show Me The Money:Access Issues: An Evaluation of the Note Teller 2 Money Identifier. AFB AccessWorld: Technology and People Who Are Blind or Visually Impaired, Vol. 8, no. 3. 2007. 